\title{
Excess Body Weight and Gastrointestinal Cancer Risk
}

\author{
Hans Scherübl \\ Klinik für Innere Medizin II, Gastroenterologie, GI Onkologie, Diabetologie und Infektiologie, \\ Vivantes Klinikum Am Urban, Berlin, Germany
}

\section{Keywords}

Colorectal cancer - Esophageal cancer - Gastric cancer . Pancreatic cancer - Biliary cancer - Gall bladder cancer . Hepatocellular carcinoma · Neoplasm · Tumor .

Malignancy · Overweight · Obesity · Adiposity

\begin{abstract}
Background: Excess body weight (EBW), defined as a body mass index $(\mathrm{BMI}) \geq 25 \mathrm{~kg} / \mathrm{m}^{2}$, has become the second most important as well as a potentially modifiable risk factor for cancer in many industrialized countries. The more excess weight people have, the higher the risk of certain cancers. Over the past several decades, EBW has been increasing globally not only among adults, but also among children and adolescents. Summary: EBW is causally associated with colorectal, esophageal (adenocarcinoma), gastric (cardia), pancreatic, biliary and hepatocellular cancer. EBW when combined with tobacco smoking, risky alcohol use, or diabetes can act synergistically to cause gastrointestinal cancer. In recent years, more and more young adults (20-40 years old) were diagnosed with EBW-associated neoplasms. People with EBW should be encouraged to join cancer screening programs. Key Messages: Keeping a healthy weight is a major public health concern and reduces the risk of cancer.
\end{abstract}

(C) 2021 The Author(s)

Published by S. Karger AG, Basel

\section{Introduction}

Among adults aged 35-70 years, cancer is the major cause of death in high-income countries. In these countries, deaths from cancer outnumber those from cardiovascular disease more than twice among 35- to 70-year- olds [1]. The lifetime cancer risk has risen overall to around 50\% in many industrialized countries.

Over the past decade, cancers associated with excess body weight (EBW) have shown troubling signs of increase among young adults and even adolescents (Fig. 1) $[2,3]$. The prevalence of EBW (BMI $\geq 25.0 \mathrm{~kg} / \mathrm{m}^{2}$ ) has expanded dramatically in almost all developing as well as developed countries, nowadays reaching pandemic levels of $60-70 \%$ of the adult population in industrialized countries. In 2016, an estimated 1.97 billion adults and over 338 million children and adolescents around the world were categorized as overweight (BMI $25.0-29.9 \mathrm{~kg} / \mathrm{m}^{2}$ ) or obese (BMI $\geq 30 \mathrm{~kg} / \mathrm{m}^{2}$ ). Between 1975 and 2016, the global number of obese adults increased 6-fold and that of obese children and adolescents more than 10-fold [4]. According to the CDC, about $40 \%$ of young US-American adults aged 20-39 years were obese in 2017 and 2018. Growing evidence supports an association between childhood or adolescent obesity and increased risk of colorectal, endometrial, kidney and pancreatic cancers and multiple myeloma in adulthood [5].

EBW is associated with colorectal, esophageal (adenocarcinoma), gastric (cardia), pancreatic, biliary, hepatocellular, kidney, thyroid, breast (postmenopausal), endometrial and ovarian cancer as well as multiple myeloma and meningioma. Anderson et al. [6] estimated that depending on gender and site, $4-38 \%$ of those malignancies could be due to EBW.

Globally, the burden of cancer attributable to obesity, expressed as the population attributable fraction (PAF), has been calculated by Avgerinos et al. [7] as 11.9\% among men and $13.1 \%$ among women. Islami et al. [8] reported that in the USA, in 2014 EBW accounted for 36\% of gallbladder and $34 \%$ of liver cancers as well as for $32 \%$ of

\section{karger@karger.com} www.karger.com/vis

Karger ${ }^{\prime \prime}=$

BOPEN ACCESS (c) 2021 The Author(s)

Published by S. Karger AG, Basel

This is an Open Access article licensed under the Creative Commons Attribution-NonCommercial-4.0 International License (CC BY-NC) (http://www.karger.com/Services/OpenAccessLicense), applicable to the online version of the article only. Usage and distribution for commercial purposes requires written permission.
Correspondence to:

Hans Scherübl, hans.scheruebl@vivantes.de 


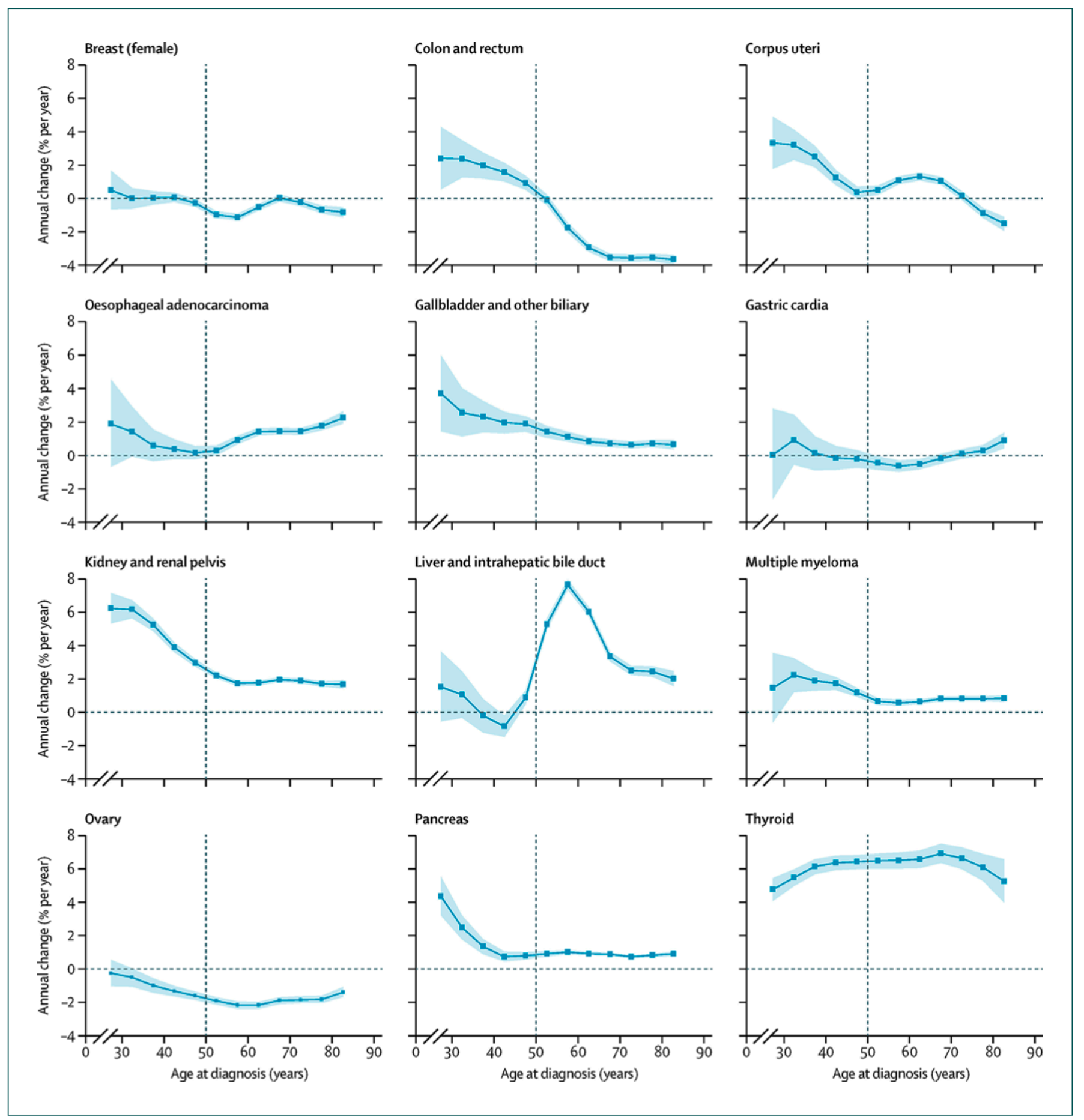

Fig. 1. Age-specific annual percent change in incidence for 12 obesity-associated cancers, 1995-2014. Dots and shaded areas represent the net annual percentage changes (in \% per year) and 95\% CIs in incidence from the age period cohort models for 12 age groups (5-year increments from age 25 years). Reprinted with permission from Elsevier [2].

esophageal, $17.5 \%$ of gastric and $17 \%$ of pancreatic cancers in adults aged 30 years and older. There is a positive dose-response association between EBW and the risk of those cancers (Fig. 2). Effective measures to permanently reduce excess weight may decrease EBW-associated cancer risk [9].

\section{EBW-Associated Carcinogenesis}

Biological mechanisms linking excess body fat to cancer encompass insulin resistance, abnormalities of the insulin growth factor (IGF)-I system and signaling, sex hormone biosynthesis and pathway, low-grade chronic inflamma- 


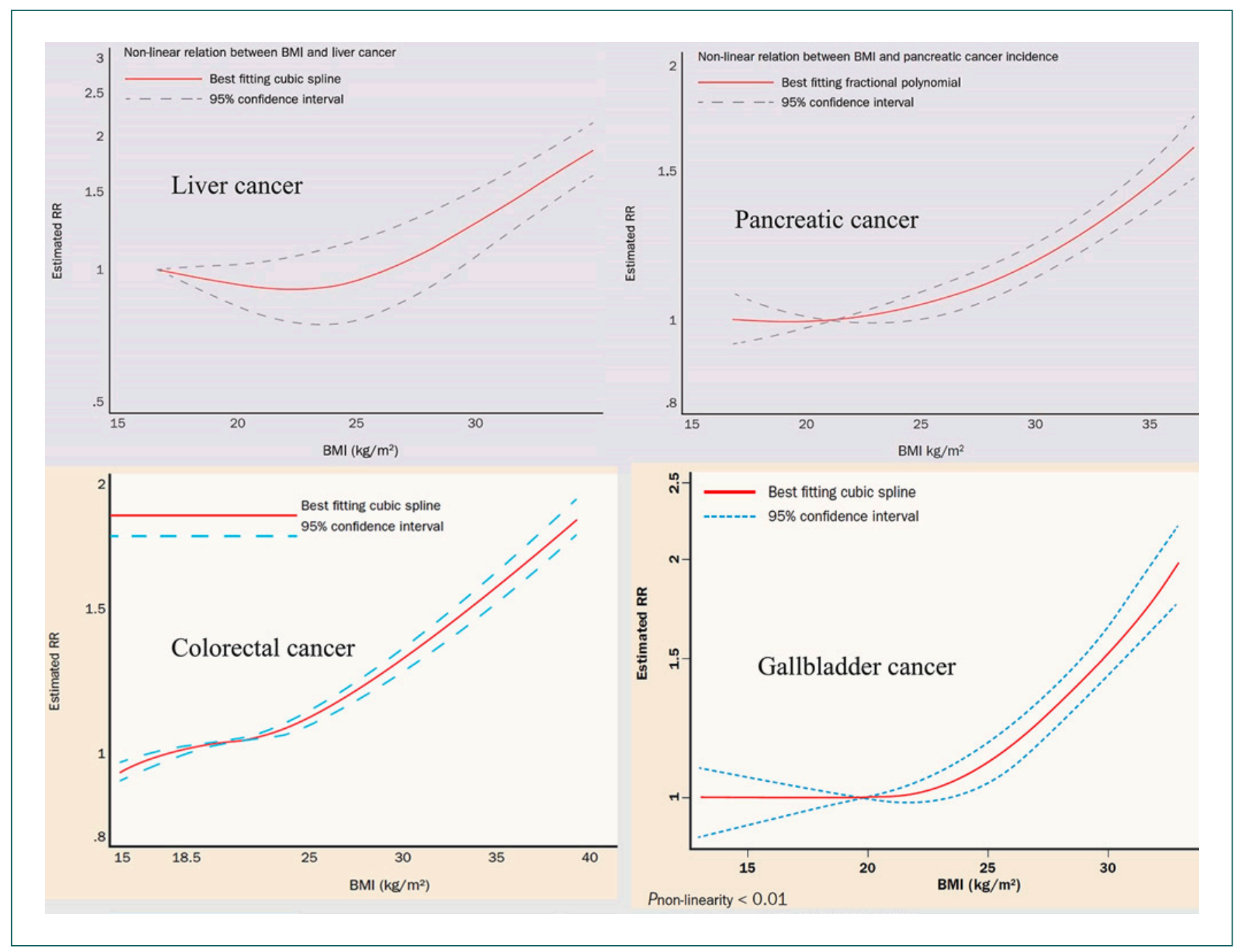

Fig. 2. Nonlinear dose-response associations between excess body weight and risks of hepatocellular (liver), pancreatic, colorectal and gallbladder cancer. Estimated relative cancer risks (RR) are shown. BMI, body mass index. This material has been reproduced with permission from the World Cancer Research Fund/American Institute for Cancer Research [11].

tion, and oxidative stress, as well as alterations in adipokine pathophysiology and the intestinal microbiome [7]. A caloric intake larger than caloric expenditure results in EBW and associated alterations in metabolism such as increased insulin levels and altered bioavailability of steroids and IGF-I. Adipose tissue is an integral hub of various hormones (adipokines), growth factors, and inflammatory cytokines such as tumor necrosis factor- $\alpha$, interleukin- 6 (IL6 ), and others. The increased level of IL-6 in obesity stimulates the liver to synthesize and secrete $\mathrm{C}$-reactive protein. Those metabolic alterations can induce both general and tumor-type-specific carcinogenic effects.

High levels of IGF-I or insulin (hyperinsulinemia) not only stimulate tumor growth in vitro, but also enhance growth of both colon and breast cancer in animals. Prospective studies provided evidence that patients with insulin resistance and higher serum levels of C-reactive protein are at increased risk of colorectal, pancreatic, endometrial and (postmenopausal) breast cancer. Similarly, higher serum levels of IGF-I go along with an increased risk of colorectal and breast cancer.

In postmenopausal women, endogenous estrogens are mainly produced by adipose tissue. The increased risk of postmenopausal breast cancer among obese women is thought to be due to the enzymatic aromatase activity of adipose tissue. Aromatase stimulates the conversion of androgens into estrogens. Alterations in endogenous hormone metabolism are considered to be the link between obesity and endometrial cancer risk.

Obesity predisposes to a proinflammatory state via increased inflammatory mediators such as IL- 6 and tumor necrosis factor- $\alpha$, as well as reduced levels of adiponectin, which has an anti-inflammatory function. In fact, obesity is considered as a chronic low-grade, systemic inflamma- 
tory state. High maximum levels of serum C-reactive protein predict a greater risk of death among patients with solid tumors [10].

\section{Colorectal Cancer}

There is a nonlinear dose-response association between EBW and colorectal cancer (CRC) risk (Fig. 2) [11]. The $\mathrm{CRC}$ risk increases by $5 \%$ per $5 \mathrm{~kg} / \mathrm{m}^{2} \mathrm{EBW}$ [11]. Nowadays, fewer people aged 50 years and older are being diagnosed with CRC in Western countries, since more of them are having colonoscopies. It is a different story for younger adults though. In Europe, between 2004 and 2016, CRC rates have increased by $1.6 \%$ per year among people in their 40 s, by $4.9 \%$ annually among 30 - to 39 -year-olds, and by even $7.9 \%$ annually among 20 - to 29-year-olds [3]. Many scientists blame the EBW pandemic [2], while others refer to an unhealthy diet, physical inactivity, altered intestinal microbiota, diabetes mellitus, risky alcohol use, and sedentary lifestyle. In a cohort study of 1,794,570 Jewish adolescents, EBW in adolescence was associated with an increased risk of subsequent colon cancers, whereas only obesity was associated with rectal cancer [5].

Due to the epidemiological increase in early-onset CRC (age $<50$ years), the American Cancer Society released an updated guideline for CRC screening in 2018. The new US-American recommendations say CRC screening should begin at a younger age, that is, at the age of 45 years for people at average risk [12]. Future screening recommendations are expected to become more personalized, aiming at precision cancer screening. The riskadapted starting age for regular screening for sporadic CRC may differ for different lifestyles and (nonhereditary) personal risk stratification. In addition to genetic predisposition (i.e., hereditary CRC), EBW, risky alcohol use, tobacco smoking, poor diet, physical inactivity, chronic inflammatory bowel disease, family history, and diabetes mellitus are well-known risk factors for CRC. Some authors favor the development of precision (i.e., risk-adapted) algorithms for screening decisions.

\section{Type 2 Diabetes, EBW, and CRC}

Cancer has overtaken both infections and cardiovascular disease as the commonest cause of death with type 2 diabetes both in Japan and in Scotland [13, 14]. Type 2 diabetes is associated with increased CRC risk. The association may partly be due to risk factors shared between the two diseases, such as EBW, aging, diet, and physical inactivity. Well-studied mechanisms for a direct link between diabetes and CRC include hyperinsulinemia, high IGF-I levels, hyperglycemia, and inflammation. In Western countries, $80-90 \%$ of adults with type 2 diabetes are overweight or obese. A nationwide Swedish cohort study has recently provided evidence that (type 2) diabetes is associated with increased CRC risk at a magnitude similar to having a family history of CRC [15]. Novel evidence argues for risk-adapted starting ages for CRC screening among patients with (type 2) diabetes and EBW. People with combined risk factors, such as family history plus type 2 diabetes/EBW, face a far higher risk of early-onset CRC than the average population [16].

\section{Pancreatic Cancer}

The world's deadliest major cancer, pancreatic cancer, has a 5 -year survival rate of around $9 \%$. Its incidence is predicted to increase and in 2030 to become the second most common cause of cancer-related death in the USA. Recognized risk factors for the development of pancreatic adenocarcinoma are EBW, tobacco smoking, diabetes, chronic pancreatitis, Helicobacter pylori infection, alcohol use ( $>45 \mathrm{~g} /$ day), and a family history of pancreatic cancer $[8,17]$.

Over the past 25 years, pancreatic adenocarcinoma has shown some of the most troubling signs of increase among young US-American adults. From 1995 to 2014, the average annual percent change in pancreatic cancer incidence increased with decreasing age from $0.77 \%(95 \%$ CI $0.57-0.98$ ) for the ages of 45-49 years to $2.47 \%$ (1.773.8 ) for the ages of 30-34 years and 4.34\% (3.19-5.50) for the ages of 25-29 years [2]. EBW is one of the most important, potentially modifiable risk factors for pancreatic cancer, with a PAF of $16.9 \%$ [8]. Pancreatic cancer risk increases by $10 \%$ per $5 \mathrm{~kg} / \mathrm{m}^{2} \mathrm{EBW}$ [11].

There are multiple interactions between EBW, tobacco smoking, alcohol use, and diabetes to cause pancreatic cancer. The risk for pancreatic cancer may be reduced by $25 \%$ or more by modifying lifestyle risk factors $[8,18,19]$. As EBW, alcohol use, tobacco smoking, and diabetes are prevalent among US adolescents, EBW combined with either risky alcohol intake, tobacco smoking, or diabetes may have contributed to the recent increases in incidence of (very-)early-onset (age $<40$ years) pancreatic adenocarcinoma $[2,20]$. Unfortunately, there are no effective means available for the early detection of asymptomatic, early-stage pancreatic adenocarcinoma in the average population. Today, primary prevention is the best option to significantly reduce the burden of pancreatic cancer deaths [18].

\section{Gallbladder and Biliary Duct Cancer}

EBW is a major, potentially modifiable risk factor for gallbladder cancer, with a PAF of $35.5 \%$ [8]. There is a nonlinear dose-response relation between EBW and gall- 
bladder cancer risk (Fig. 2). Cancer risk increases by $25 \%$ per $5 \mathrm{~kg} / \mathrm{m}^{2} \mathrm{EBW}$ [11]. In the last 30 years, the prevalence of pediatric and adolescent gallstones, the primary risk factor for gallbladder cancer in adults, has increased in Western countries. An approximately linear relationship exists between body weight and gallstone development. Up to 4 out of 5 people with gallbladder cancer have gallstones when they are diagnosed. Gallstones and gallbladder inflammation are important risk factors for gallbladder cancer [21]. Compared with nondiabetic individuals, both men and women with type 2 diabetes have an increased risk of gallbladder cancer [22]. There is also strong evidence for an association between EBW, type 2 diabetes, and excess risk of intrahepatic cholangiocarcinoma [23].

\section{Liver Cancer (Hepatocellular Carcinoma)}

Nonalcoholic fatty liver disease (NAFLD), also known as metabolic (dysfunction) associated fatty liver disease, is the most common liver disorder in the world, in particular in Western countries, where the main risk factors for NAFLD, EBW, and type 2 diabetes mellitus are very common. Globally, NAFLD has a reported prevalence of 6-35\% (median 20\%). NAFLD may progress to nonalcoholic steatohepatitis (NASH), and NASH to liver cirrhosis and liver cancer (hepatocellular carcinoma [HCC]). EBW is the most important, potentially modifiable risk factor for HCC, with a PAF of $33.9 \%$ in the USA [8]. A positive nonlinear dose-response relation exists between EBW and HCC risk (Fig. 2). HCC risk increases by $30 \%$ per $5 \mathrm{~kg} / \mathrm{m}^{2}$ EBW [11]. Not only EBW, but also risky alcohol use is prevalent in Europe. Alcohol intake can synergistically enhance the obesity-associated HCC risk [24]. Moreover, EBW can further increase the HCC risk among people with chronic hepatitis $\mathrm{B}$ or $\mathrm{C}$ infections or with hemochromatosis [25]. Combining EBW with smoking tobacco similarly leads to an increased cumulative HCC risk [26]. In addition, there is now robust evidence of an association between type 2 diabetes and an increased $\mathrm{HCC}$ risk [27]. EBW-induced NASH interacts additively or even synergistically with other major HCC risk factors, such as chronic infection with hepatitis B and hepatitis C, alcohol drinking, tobacco smoking, and diabetes, to cause HCC. HCC is the major cause of liver-specific death among patients with liver cirrhosis. Early detection of asymptomatic HCC by liver ultrasound every 6 months should be offered to patients with liver cirrhosis. Sixmonthly ultrasound scanning can not only detect earlystage HCC but also asymptomatic, early-stage intrahepatic cholangiocellular carcinoma in patients with liver cirrhosis.

\section{Esophageal, Esophagogastric Junction and Gastric Cancer}

The incidence of esophageal adenocarcinoma (EA) and esophagogastric junction adenocarcinoma is increasing, and this has been attributed to the increased prevalence of Barrett's esophagus, gastroesophageal reflux disease, EBW, and diabetes. EBW is a major, potentially modifiable risk factor for EA, with a PAF of $32.2 \%$ in the USA [8]. Doseresponse analysis showed a statistically significant $48 \%$ increased risk of EA per $5 \mathrm{~kg} / \mathrm{m}^{2}$ EBW [11]. Patients with type 2 diabetes mellitus have a higher prevalence of EA, esophagogastric junction adenocarcinoma, and gastric cancer $[28,29]$. There is a positive dose-response relationship between EBW and cardia cancer [11,30]. Dose-response analysis demonstrated a statistically significant $23 \%$ increased risk of cardia cancer per $5 \mathrm{~kg} / \mathrm{m}^{2} \mathrm{EBW}$ [11]. EBW is a major, potentially modifiable risk factor for gastric cancer as well, with a PAF of $17.5 \%$ in the USA [8].

In the USA, the incidence of early-onset gastric cancer has been steadily increasing since 1995, comprising more than $30 \%$ of new gastric cancer cases today. Early-onset gastric cancer shows worrisome trends and oncogenic features [31]. The Korean National Cancer Screening Program provided strong evidence for screening gastroscopy - starting at the age of 40 years - to effectively reduce both gastric cancer and overall mortality [32]. In Western countries, screening esophagogastroscopy is generally restricted to high-risk populations.

Important risk factors for gastric cancer are H. pylori infection, dietary (nitroso compounds, high-salt diet with few vegetables) and lifestyle factors (EBW, tobacco smoking, risky alcohol use), family history, male sex, diabetes mellitus, atrophic gastritis, and intestinal metaplasia. Risk factors for (either squamous or glandular cell) esophageal cancer comprise tobacco smoking, alcohol intake, family history, (previous) head and neck cancer, male sex, EBW, Barrett's esophagus, reflux disease, and diabetes mellitus. With 3 or more of those risk factors being present concomitantly, overweight or obese people should consider undergoing screening esophagogastroscopy at the age of 40 years to detect either early esophageal cancer, early esophagogastric junction adenocarcinoma, early gastric cancer, or precancerous lesions. In the future, biomarkers, liquid biopsy, microbial signatures, and metabolomic profiles will optimize risk stratification and may well lead to precision cancer screening [33].

\section{Cancer Prevention - Preventive Oncology}

There is no single or simple solution to the EBW pandemic. It is a complex problem and there has to be a multifaceted approach. Education to a healthy lifestyle, calor- 
ic restriction, physical exercise, healthy diet, and even bariatric surgery are mainstays. EBW is not the only potentially modifiable risk factor for gastrointestinal cancers. More than $45 \%$ of gastrointestinal cancers are potentially preventable $[8,34]$. Effective cancer prevention programs can significantly reduce the burden of gastrointestinal cancer deaths. There is considerable need for action.

\section{Conflict of Interest Statement}

The author has no conflicts of interest to declare.

\section{Funding Sources}

The author received no funding.

\section{References}

1 Dagenais GR, Leong DP, Rangarajan S, Lanas F, Lopez-Jaramillo P, Gupta R, et al. Variations in common diseases, hospital admissions, and deaths in middle-aged adults in 21 countries from five continents (PURE): a prospective cohort study. Lancet. 2020 Mar; 395(10226):785-94.

2 Sung H, Siegel RL, Rosenberg PS, Jemal A. Emerging cancer trends among young adults in the USA: analysis of a population-based cancer registry. Lancet Public Health. 2019 Mar;4(3):e137-47.

3 Vuik FE, Nieuwenburg SA, Bardou M, Lansdorp-Vogelaar I, Dinis-Ribeiro M, Bento MJ, et al. Increasing incidence of colorectal cancer in young adults in Europe over the last 25 years. Gut. 2019 Oct;68(10):1820-6.

4 Sung H, Siegel RL, Torre LA, Pearson-Stuttard J, Islami F, Fedewa SA, et al. Global patterns in excess body weight and the associated cancer burden. CA Cancer J Clin. 2019 Mar; 69(2):88-112

5 Levi Z, Kark JD, Katz LH, Twig G, Derazne E, Tzur D, et al. Adolescent body mass index and risk of colon and rectal cancer in a cohort of 1.79 million Israeli men and women: a population-based study. Cancer. 2017 Oct;123(20): 4022-30.

6 Anderson AS, Key TJ, Norat T, Scoccianti C, Cecchini M, Berrino F, et al. European Code Against Cancer 4th Edition: obesity, body fatness and cancer. Cancer Epidemiol. 2015; 39(Suppl 1):S34-45.

7 Avgerinos KI, Spyrou N, Mantzoros CS, Dalamaga $\mathrm{M}$. Obesity and cancer risk: emerging biological mechanisms and perspectives. Metabolism. 2019 Mar;92:121-35.

8 Islami F, Goding Sauer A, Miller KD, Siegel RL, Fedewa SA, Jacobs EJ, et al. Proportion and number of cancer cases and deaths attributable to potentially modifiable risk factors in the United States. CA Cancer J Clin. 2018 Jan; 68(1):31-54.

9 Schauer DP, Feigelson HS, Koebnick C, Caan B, Weinmann S, Leonard AC, et al. Bariatric Surgery and the Risk of Cancer in a Large Multisite Cohort. Ann Surg. 2019 Jan;269(1): 95-101.

10 Shrotriya S, Walsh D, Nowacki AS, Lorton C, Aktas A, Hullihen B, et al. Serum C-reactive protein is an important and powerful prognostic biomarker in most adult solid tumors. PLoS One. 2018 Aug;13(8):e0202555.

11 World Cancer Research Fund/American Institute for Cancer Research. Diet, Nutrition, Physical Activity and Cancer: A Global Perspective. The Third Expert Report. Available from: www.wcrf.org/dietandcancer.
12 American Cancer Society Updates Colorectal Cancer Screening Guideline. Available from: https://www.cancer.org/latest-news/american-cancer-society-updates-colorectal-cancer-screening-guideline.html.

13 Nakamura J, Kamiya H, Haneda M, Inagaki N, Tanizawa Y, Araki E, et al. Causes of death in Japanese patients with diabetes based on the results of a survey of 45,708 cases during 2001-2010: report of the Committee on Causes of Death in Diabetes Mellitus. J Diabetes Investig. 2017 May;8(3):397-410.

14 Collier A, Meney C, Hair M, Cameron L, Boyle JG. Cancer has overtaken cardiovascular disease as the commonest cause of death in Scottish type 2 diabetes patients: a population-based study (The Ayrshire Diabetes Follow-up Cohort study). J Diabetes Investig. 2020 Jan;11(1):55-61.

15 Ali Khan U, Fallah M, Tian Y, Sundquist K, Sundquist J, Brenner H, et al. Personal History of Diabetes as Important as Family History of Colorectal Cancer for Risk of Colorectal Cancer: A Nationwide Cohort Study. Am J Gastroenterol. 2020 Jul;115(7):1103-9.

16 Ali Khan U, Fallah M, Sundquist K, Sundquist J, Brenner H, Kharazmi E. Risk of colorectal cancer in patients with diabetes mellitus: a Swedish nationwide cohort study. PLoS Med. 2020 Nov; 17(11):e1003431.

17 Rawla P, Sunkara T, Gaduputi V. Epidemiology of Pancreatic Cancer: Global Trends, Etiology and Risk Factors. World J Oncol. 2019 Feb;10(1):10-27.

18 Jiao L, Mitrou PN, Reedy J, Graubard BI, Hollenbeck AR, Schatzkin A, et al. A combined healthy lifestyle score and risk of pancreatic cancer in a large cohort study. Arch Intern Med. 2009 Apr;169(8):764-70.

19 Parkin DM, Boyd L, Walker LC. 16. The fraction of cancer attributable to lifestyle and environmental factors in the UK in 2010. Br J Cancer. 2011 Dec;105(Suppl 2):S77-81.

20 McWilliams RR, Maisonneuve P, Bamlet WR, Petersen GM, Li D, Risch HA, et al. Risk factors for early-onset and very-early-onset pancreatic adenocarcinoma: a pancreatic cancer case-control consortium (PanC4) analysis. Pancreas. 2016 Feb;45(2):311-6.

21 Wang J, Xu C, Cheng Q, Zhao J, Wu S, Li W, et al. RNA Sequencing Revealed Signals of Evolution from Gallbladder Stone to Gallbladder Carcinoma. Front Oncol. 2020 May; 10:823.

22 Gu J, Yan S, Wang B, Shen F, Cao H, Fan J, et al. Type 2 diabetes mellitus and risk of gallbladder cancer: a systematic review and metaanalysis of observational studies. Diabetes Metab Res Rev. 2016 Jan;32(1):63-72.
23 Petrick JL, Thistle JE, Zeleniuch-Jacquotte A, Zhang X, Wactawski-Wende J, Van Dyke AL, et al. Body Mass Index, Diabetes and Intrahepatic Cholangiocarcinoma Risk: The Liver Cancer Pooling Project and Meta-analysis. Am J Gastroenterol. 2018 Oct;113(10):1494-505.

24 Loomba R, Yang HI, Su J, Brenner D, BarrettConnor E, Iloeje U, et al. Synergism between obesity and alcohol in increasing the risk of hepatocellular carcinoma: a prospective cohort study. Am J Epidemiol. 2013 Feb;177(4): 333-42.

25 Yu MW, Lin CL, Liu CJ, Yang SH, Tseng YL, $\mathrm{Wu}$ CF. Influence of metabolic risk factors on risk of hepatocellular carcinoma and liver-related death in men with chronic hepatitis B: a large cohort study. Gastroenterology. 2017 Oct;153(4):1006-1017.e5.

26 Meyer J, Rohrmann S, Bopp M, Faeh D; Swiss National Cohort Study Group. Swiss National cohort study group. Impact of smoking and excess body weight on overall and site-specific cancer mortality risk. Cancer Epidemiol Biomarkers Prev. 2015 Oct;24(10):1516-22.

27 Baecker A, Liu X, La Vecchia C, Zhang ZF. Worldwide incidence of hepatocellular carcinoma cases attributable to major risk factors. Eur J Cancer Prev. 2018 May;27(3):205-12.

28 Petrick JL, Li N, Anderson LA, Bernstein L, Corley DA, El-Serag HB, et al. Diabetes in relation to Barrett's esophagus and adenocarcinomas of the esophagus: A pooled study from the International Barrett's and Esophageal Adenocarcinoma Consortium. Cancer. 2019 Dec;125(23):4210-23.

29 Yang HJ, Kang D, Chang Y, Ahn J, Ryu S, Cho $\mathrm{J}$, et al. Diabetes mellitus is associated with an increased risk of gastric cancer: a cohort study. Gastric Cancer. 2020 May;23(3):382-90.

30 Lin XJ, Wang CP, Liu XD, Yan KK, Li S, Bao $\mathrm{HH}$, et al. Body mass index and risk of gastric cancer: a meta-analysis. Jpn J Clin Oncol. 2014 Sep;44(9):783-91.

31 Bergquist JR, Leiting JL, Habermann EB, Cleary SP, Kendrick ML, Smoot RL, et al. Early-onset gastric cancer is a distinct disease with worrisome trends and oncogenic features. Surgery. 2019 Oct;166(4):547-55.

32 Jun JK, Choi KS, Lee HY, Suh M, Park B, Song $\mathrm{SH}$, et al. Effectiveness of the Korean National Cancer Screening Program in reducing gastric cancer mortality. Gastroenterology. 2017 May;152(6):1319-28.e7.

33 Huang S, Guo Y, Li Z, Zhang Y, Zhou T, You $\mathrm{W}$, et al. A systematic review of metabolomic profiling of gastric cancer and esophageal cancer. Cancer Biol Med. 2020 Feb;17(1):181-98.

34 Scherübl H. Alcohol use and gastrointestinal cancer risk. Visc Med. 2020 Jun;36(3):175-81. 